\title{
Caracterización anatómica del conducto nasopalatino mediante tomografía computarizada de haz cónico en una población peruana
}

\author{
Anatomic characterization of the nasopalatine canal through cone beam computed tomography in a Peruvian \\ population
}

Jorge Luis Obando Castillo ${ }^{1, a ; 2 b}$, Vilma Elizabeth Ruiz García de Chacón²,c,

\section{RESUMEN}

El Conducto Nasopalatino (CNP) es una estructura importante que puede ser susceptible de daño durante procedimientos quirúrgicos en el maxilar superior anterior; por lo tanto examinar meticulosamente la anatomía y variantes del CNP se hace imperativo para un buen diagnóstico y un apropiado plan de tratamiento. Objetivo: Realizar una caracterización anatómica del CNP utilizando Tomografía Computarizada de Haz Cónico (TCHC). Material y métodos: Se evaluaron 154 imágenes de TCHC, en las que se analizó CNP en cuanto a su longitud, forma, e inclinación con respecto al paladar duro y el diámetro anteroposterior del foramen incisivo (FI) en hombres y mujeres de 18 años de edad en adelante. Resultados: A la evaluación del CNP en cortes sagitales de TCHC presentó diferentes formas; se encontró que la forma más común fue la cilíndrica en 89 pacientes (58\%), seguida de la forma de embudo en 38 (25\%), y las menos frecuentes fueron la forma a de reloj de arena en 20 $(13 \%)$ y de huso en 7 (5\%). La longitud promedio del CNP fue de $10,83 \mathrm{~mm}( \pm 2,13)$, el promedio del diámetro anteroposterior del FI fue de $3,44 \mathrm{~mm}( \pm 0,80)$ y la angulación promedio en relación al paladar duro fue de $16,44^{\circ}( \pm 7,32)$.Conclusiones: El estudio mostró la variabilidad del CNP en la población peruana, tanto en la forma, longitud e inclinación, así como también en el diámetro del FI.

PALABRAS CLAVE: Maxilar, tomografía computarizada de haz cónico, variación anatómica, conducto nasopalatino, foramen incisivo.

\begin{abstract}
SUMMARY
Nasopalatine Canal (NPC) is an important structure that may be susceptible to damage during surgical procedures in the anterior maxilla, therefore a thorough examination of the anatomy and variants of the NPC is imperative for a good diagnosis and an appropriate treatment plan. Objective: To perform an anatomic characterization of NPC using Computed Beam Computed Tomography (CBCT). Material and methods: 154 images of CBCT were evaluated, in which the NPC was analyzed in terms of length, shape, and inclination with respect to the hard palate and the maximum anteroposterior of the incisive foramen (IF) in men and women of 18 years of age and onwards. Results: to the evaluation of the NCP in sagittal slices of CBCT, it presented different forms, it was found to the cylindrical shape as the most common in 89 patients (58\%), followed by the funnel shape in 38 $(25 \%)$, and the less frequent were the shape of hourglass in $20(13 \%)$ and spindle $(5 \%)$. The average length of the NCP was $10.83 \mathrm{~mm}( \pm 2.13)$, the average of the anteroposterior diameter of the FI was $3.44 \mathrm{~mm}( \pm 0.80)$ and the average angulation of the CNP in relation to the hard palate was $16.44^{\circ}( \pm 7.32)$. Conclusions: The study showed the variability of NCP in the Peruvian population, like in shape, length and inclination, as well as the diameter of the FI.
\end{abstract}

KEY WORDS: Maxilla, cone-beam computed tomography, anatomic variation, nasopalatine canal, incisive foramen.

Facultad de Odontología. Universidad de Antioquia. Medellín, Colombia.

Especialidad en Radiología Bucal y Máxilofacial, Facultad de Estomatología, Universidad Peruana Cayetano Heredia. Lima, Perú.

Cirujano Dentista, ${ }^{\mathrm{b}}$ Residente; ${ }^{\mathrm{c}}$ Docente; ${ }^{\mathrm{a}}$ Magister Especialista Radiología Bucal y Máxilofacial. 


\section{INTRODUCCION}

El Conducto Nasopalatino (CNP) también conocido como canal incisivo o canal palatino anterior, es un pasaje delgado presente en la línea media del maxilar anterior que conecta el paladar con piso de la cavidad nasal (1)Y, and Z. El CNP se abre en la cavidad oral a través del agujero o foramen incisivo (FI), generalmente debajo de la papila incisiva a $4-5 \mathrm{~mm}$ posterior a los dientes incisivos centrales; en la cavidad nasal se continúa como forámenes de Stenson generalmente 2 en número ( $1 \mathrm{Y}$, and $\mathrm{Z}, 2)$. A través de estos conductos pasan el nervio y la arteria nasopalatinos que suministran la región palatina anterior $(3,4)$ morphology and dimensions of the nasopalatine canal on two-dimensional and three-dimensional (2D and 3D. Un estudio histológico realizado en el año 2009 mostró que el CNP contenía una gran arteria rodeada de venas y nervios mielinados (nervio nasopalatino) e incluso fueron observadas pequeñas glándulas seromucosas (5).

Se han reportado dificultades con respecto a la anatomía del CNP durante procedimientos dentales y quirúrgicos en el maxilar anterior como anestesia local, implantes de incisivos centrales, extracción quirúrgica de dientes supernumerarios, etc., (6). El CNP es una estructura anatómica importante que puede ser susceptible de daño durante dichos procedimientos, por tal motivo la descripción de las características y las variaciones del conducto son cruciales para optimizar la planificación quirúrgica y evitar complicaciones, previniendo secuelas originadas por el contacto con el tejido nervioso o generando disfunción sensorial $(3,5)$ morphology and dimensions of the nasopalatine canal on two-dimensional and three-dimensional (2D and 3D.

Se han realizado varios estudios para evaluar las características y determinar la variabilidad humana del CNP usando tomografías computarizadas. En cuanto a la morfología se ha encontrado que el conducto puede tener diferentes variaciones, sin embargo no está establecida o aceptada una terminología universal para ellas. Un estudio definió la forma del conducto a través de Tomografía Espiral Multicorte (TEM) en cortes coronales como morfología en "Y" o cilíndrica (4)morphology and dimensions of the nasopalatine canal on two-dimensional and three-dimensional (2D and $3 \mathrm{D}$, y otro estudio informó que el conducto en dichos cortes también podría presentarse como dos canales paralelos (7). Además el conducto ha sido clasificado en un plano sagital a través de TCHC en diferentes formas tales como: cilíndrica, embudo, reloj de arena y huso (5morphology and dimensions of the nasopalatine canal on two-dimensional and three-dimensional (2D and 3D,7,8).

Algunas investigaciones han reportado las dimensiones del CNP y mostraron que el sexo de los pacientes tuvo una influencia en la longitud y diámetro del CNP, siendo los valores medios generalmente más altos para el sexo masculino $(1,2,9,10)$. Algunos autores consideraron la inclinación del CNP en cortes sagitales de TCHC y lo han clasificado como vertical o inclinado $(1,3,11)$. Un estudio encontró que los conductos inclinados se observaron con mayor frecuencia que los verticales (1). Otras investigaciones demostraron que los pacientes parecen tener un tipo de conducto inclinado curvado $(3,11)$. El objetivo del presente estudio fue realizar una caracterización anatómica del CNP en cuanto a su forma, longitud, inclinación, orientación y el diámetro anteroposterior del FI a través de TCHC en una población peruana.

\section{MATERIAL Y MÉTODOS}

El presente estudio fue descriptivo, retrospectivo, observacional y transversal. La población estuvo constituida por TCHC de pacientes que acudieron al Servicio de Radiología Bucomaxilofacial de la Clínica Estomatológica de la Universidad Peruana Cayetano Heredia (UPCH) en la Sede San Martín de Porres en el año 2017. Al tratarse de una base de datos codificada se garantizó el anonimato de los propietarios de las tomografías. Se procedió a la recolección de datos luego de recibir la aprobación de la Unidad Integrada de Gestión de Investigación, Ciencia y Tecnología (UIGCT) y la posterior aprobación del Comité Institucional de Ética de la UPCH. La selección de las tomografías se realizó por conveniencia, eligiendo las tomografías del año 2017 que cumplieron con los criterios de selección. Al aplicar los criterios de inclusión (TCHC de hombres y mujeres mayores de 18 años, considerados como adultos por la OMS) y exclusión (TCHC que evidencien secuelas de procedimientos quirúrgicos, o injertos óseos en el maxilar anterior, presencia y/o secuelas de patología, traumatismos en el maxilar anterior o TCHC que no presenten una calidad satisfactoria de imagen) se obtuvo un tamaño maestral de 154 TCHC. 
Se utilizó el método de observación directa en donde se analizó cada volumen tomográfico con el equipo modelo CS9300 de la marca Carestream, por medio del software visualizador CS 3D Imaging, en una pantalla de 16 pulgadas de marca Lenovo y se anotaron las observaciones en fichas de recolección para cada una de las variables consignadas en la investigación. Se realizó una calibración con un especialista en Radiología Bucomaxilofacial con más de 5 años de experiencia (estándar de oro). Para la calibración se utilizó el método Estadístico de Kappa, obteniéndose un valor de 0,998 . Se realizó una prueba piloto con el $10 \%$ de la muestra (16 TCHC) para identificar la existencia de oportunidades de mejora en la recolección de datos final.

En cada volumen tomográfico se realizó un análisis dinámico para localizar el CNP. En la sección de cortes oblicuos, una vez localizado el conducto, se procedió a seguir su eje central en todos los planos para lograr un corte sagital estricto, en el cual se evaluó el tipo de morfología que presentaba, evidenciándose así cuatro formas del CNP basadas en sus paredes anteriores y posteriores: A. Forma cilíndrica: en donde las paredes fueron paralelas. B. Forma de embudo: en la cual la dimensión anteroposterior iba aumentando desde la fosa nasal al paladar duro. C. Forma de huso: en donde la dimensión anteroposterior a nivel del medio era más ancha en comparación con los niveles de las dimensiones de la fosa nasal y del paladar duro. D. Forma de reloj de arena: en la cual la dimensión anteroposterior del conducto está disminuida a nivel del medio en comparación con las dimensiones a nivel de la fosa nasal y del paladar duro. (figura 1). En el mismo corte sagital estricto, con la herramienta de medición del software se determinó la longitud del CNP y el diámetro anteroposterior del FI (figura 2). Con la herramienta de ángulo del software se determinó la inclinación del CNP en relación a una perpendicular al plano palatino (figura 3); la orientación se consideró dependiendo del resultado de la inclinación: cuando el resultado fue menor de 10 grados se consideró como vertical; y cuando el resultado fue mayor o igual a 10 grados como inclinado (1)Y, and $\mathrm{Z}$ (figura 4), Se obtuvieron resultados descriptivos que se clasificaron según morfología, longitud e inclinación del CNP y diámetro anteroposterior del FI. Posteriormente se obtuvo la frecuencia absoluta y relativa y finalmente los datos obtenidos se representaron en tablas y gráficos.
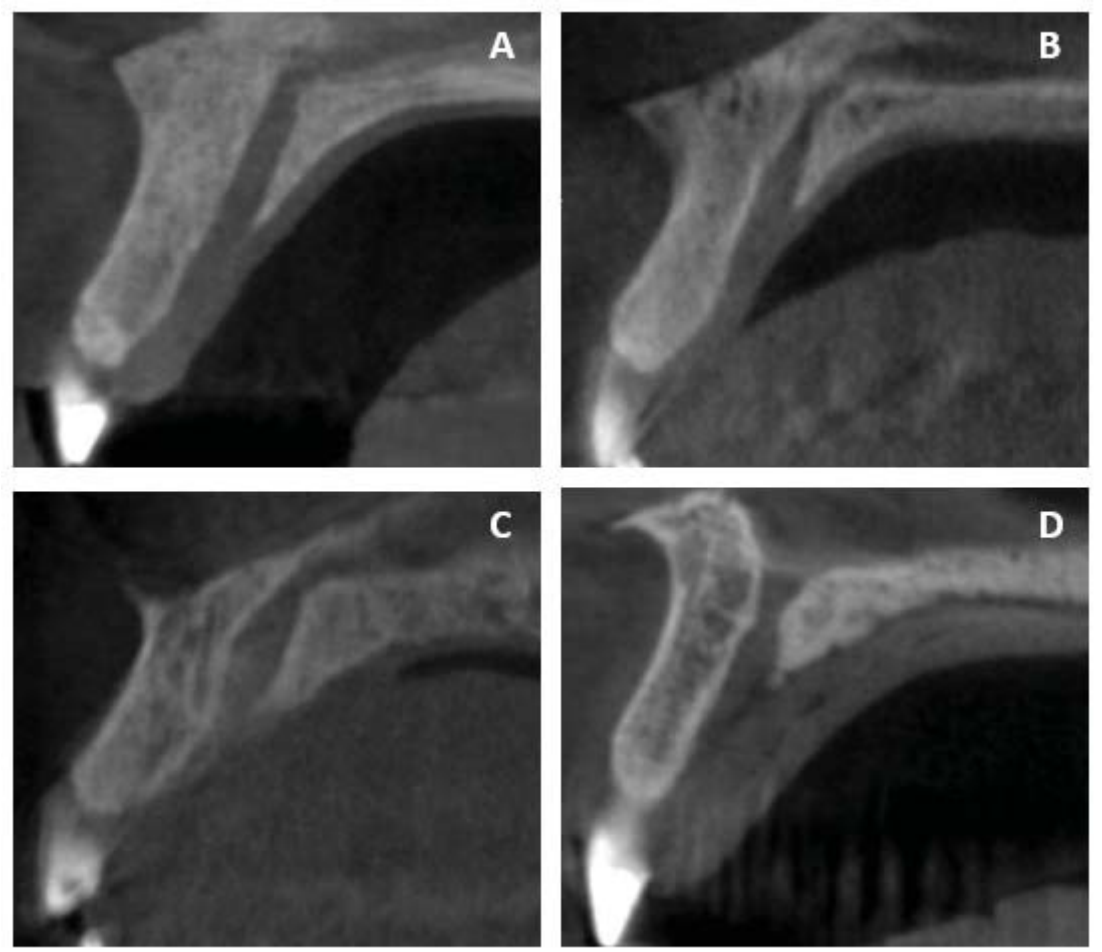

Figura 1. Imágenes de TCHC en planos sagitales tomadas de la muestra del presente estudio. Se evidenció cuatro formas del CNP basadas en las paredes anteriores y posteriores del conducto. A. Forma cilíndrica: B. Forma de embudo C. Forma de huso D. Forma de reloj de arena, 


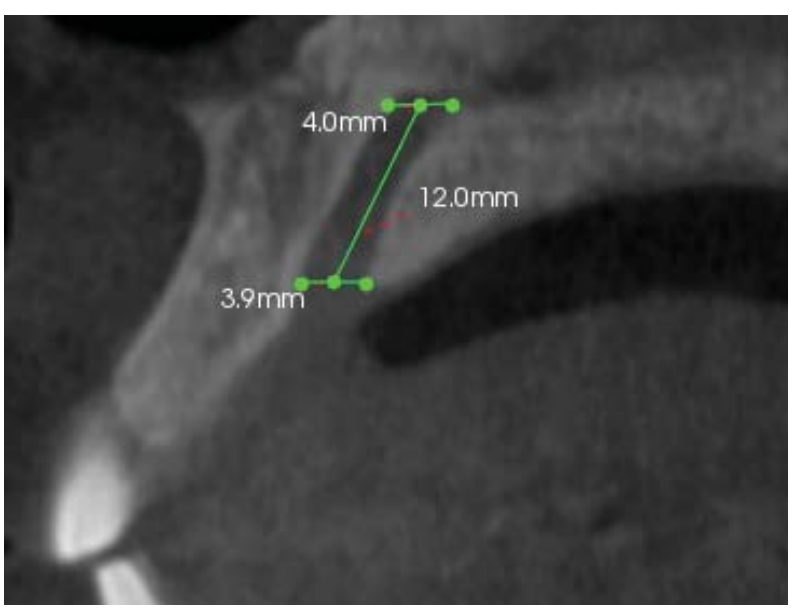

Figura 2. Imagen de TCHC en un plano sagital tomado de la muestra. Se ejemplifica el método que se usó para determinar la longitud del CNP y el diámetro anteroposterior del FI.

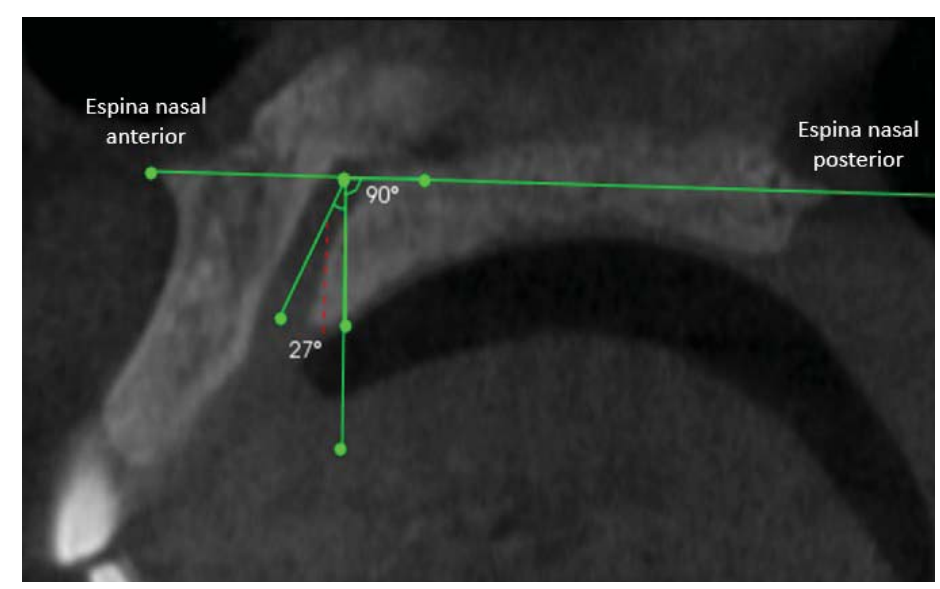

Figura 3. Imagen TCHC en un corte sagital tomada de la muestra. Se ejemplifica el método que se usó para determinar el grado de inclinación del CNP respecto a la perpendicular al plano palatino. En este caso la inclinación fue de $27^{\circ}$.
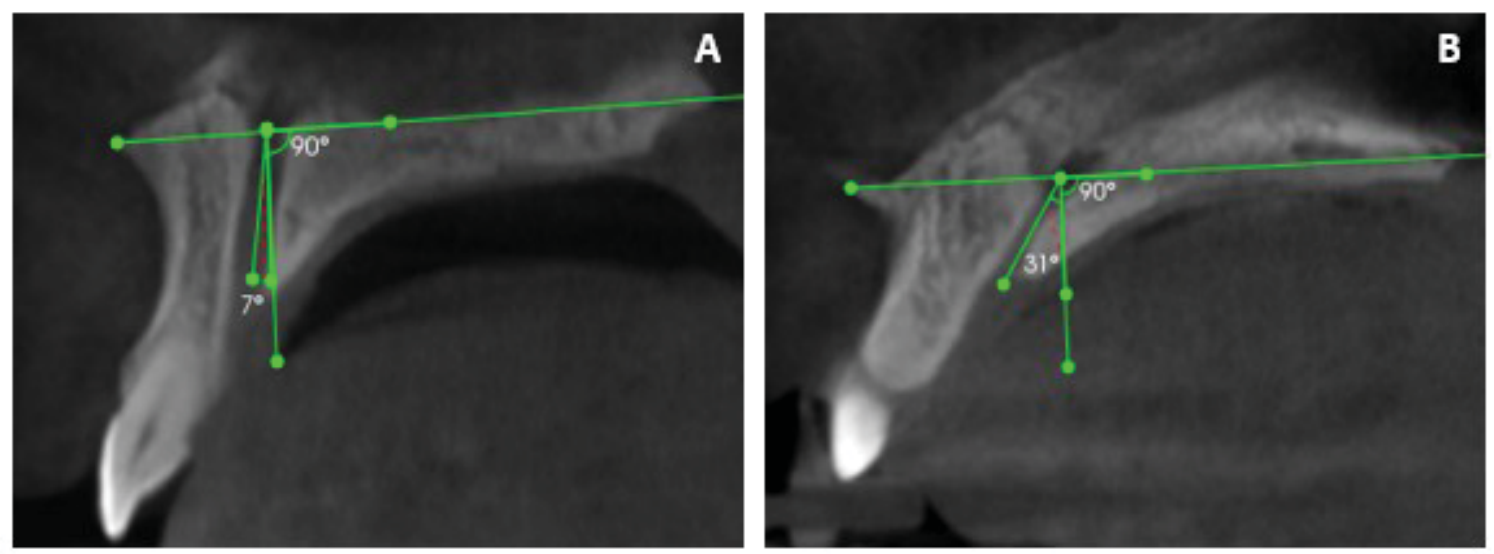

Figura 4. Imágenes de TCHC tomadas de la muestra. A. Ejemplo de un CNP de tipo vertical, cuyo ángulo entre la perpendicular al plano palatino es inferior a $10^{\circ}$. B. Ejemplo de un CNP de tipo inclinado, cuyo ángulo entre la perpendicular al plano palatino es superior a $10^{\circ}$ 


\section{RESULTADOS}

La muestra estuvo conformada por 154 TCHC del año 2017 en donde 55\% fueron de sexo femenino y $45 \%$ fueron de sexo masculino. La edad promedio general fue de 39,9 años $( \pm 17,33)$. La edad promedio para el sexo femenino fue de 41,28 años $( \pm 17,16)$, mientras que la edad promedio para el sexo masculino fue de 38,27 años $( \pm 17,53)$.

\section{Clasificación del CNP según su forma}

El CNP fue clasificado según su forma vista en los cortes sagitales como: Cilíndrico, embudo, huso o reloj de arena (1) (figura 1). La forma más comúnmente encontrada fue la cilíndrica, seguida por la forma de embudo y las formas menos encontradas fueron la de reloj de arena y de huso, los datos de los resultados se encuentran en la tabla 1 . No se observaron diferencias significativas en cuanto a sexo.

\section{Longitud del CNP}

La longitud del CNP se obtuvo midiendo a lo largo del eje del conducto desde su parte superior a nivel de la fosa nasal e inferiormente a nivel del paladar duro (figura 2). Los datos de los resultados de los rangos de longitud y la relación entre longitud y sexo se encuen- tran en la tabla 2. Se evidenció entonces un tamaño relativamente mayor en el sexo masculino respecto al femenino.

\section{Inclinación y orientación del CNP}

La inclinación del CNP fue tomado por el ángulo formado entre una perpendicular al plano palatino (línea que pasa entre la espina nasal anterior y la espina nasal posterior siguiendo la orientación el piso de las fosas nasales) y una línea paralela a la dirección del CNP (figura 3). Los datos de la distribución de la inclinación del CNP según sexo se encuentran en la tabla 3.

La orientación del CNP fue definida como vertical cuando el ángulo del conducto fue inferior a $10^{\circ} \mathrm{e}$ inclinada cuando el ángulo resultante fue igual o superó los $10^{\circ}(1) \mathrm{Y}$, and $\mathrm{Z}$. Los datos de la distribución de la orientación del CNP según sexo se encuentran en la tabla 4. En la figura 5 se muestran 2 ejemplos de orientación del CNP que tuvo la muestra.

Diámetro anteroposterior del foramen incisivo El diámetro anteroposterior del FI fue tomado como se muestra en la figura 2. Los datos del diámetro anteroposterior del FI según sexo se encuentran en la tabla 5. Se evidenció dimensiones un poco mayores en el sexo masculino respecto al femenino.

Tabla 1. Morfología del Conducto Nasopalatino

\begin{tabular}{cccccccc}
\hline \multicolumn{2}{c}{ CILINDRICA } & \multicolumn{2}{c}{ EMBUDO } & \multicolumn{2}{c}{ HUSO } & \multicolumn{2}{c}{ RELOJ DE ARENA } \\
$\mathrm{n}$ & $\%$ & $\mathrm{n}$ & $\%$ & $\mathrm{n}$ & $\%$ & $\mathrm{n}$ & $\%$ \\
\hline 89 & 58 & 38 & 25 & 7 & 5 & 20 & 13 \\
\hline
\end{tabular}

Tabla 2. Distribución de la longitud del conducto nasopalatino según sexo.

\begin{tabular}{cccccccc}
\hline $\mathrm{mm}$ & $\mathrm{DE}$ & $\begin{array}{c}\text { FEMENINO } \\
\text { Mínimo }\end{array}$ & Máximo & $\mathrm{mm}$ & $\mathrm{DE}$ & $\begin{array}{r}\text { MASCULINO } \\
\text { Mínimo }\end{array}$ & Máximo \\
\hline 9,79 & 1,95 & 5,6 & 16,5 & 12,11 & 2,15 & 7 & 19,1 \\
\hline
\end{tabular}


Tabla 3. Distribución de la inclinación del Conducto Nasopalatino según sexo.

\begin{tabular}{cccccccc}
\hline \multirow{2}{*}{ GRADOS } & \multicolumn{2}{c}{ FEMENINO } & \multicolumn{4}{c}{ MASCULINO } \\
& DE & Mínimo & Máximo & GRADOS & DE & Mínimo & Máximo \\
\hline
\end{tabular}

Tabla 4. Distribución de la orientación del Conducto Nasopalatino según sexo.

\begin{tabular}{cccccccc}
\hline & \multicolumn{2}{c}{ FEMENINO } & \multicolumn{2}{c}{ MASCULINO } \\
$\mathrm{n}$ & \multicolumn{2}{c}{ INCLINADO } & \multicolumn{2}{c}{ VERTICAL } & \multicolumn{2}{c}{ INCLINADO } & \multicolumn{2}{c}{ VERTICAL } \\
\hline 74 & $\%$ & $\mathrm{n}$ & $\%$ & $\mathrm{n}$ & $\%$ & $\mathrm{n}$ & $\%$ \\
\hline
\end{tabular}

Tabla 5. Diámetro anteroposterior del Foramen Incisivo según sexo.

\begin{tabular}{cccccccc}
\hline & \multicolumn{3}{c}{ FEMENINO } & & \multicolumn{3}{c}{ MASCULINO } \\
$\mathrm{mm}$ & $\mathrm{DE}$ & Mínimo & Máximo & $\mathrm{mm}$ & $\mathrm{DE}$ & Mínimo & Máximo \\
\hline 3,28 & 0,68 & 1,5 & 4,9 & 3,61 & 0,89 & 2,1 & 5,4 \\
\hline
\end{tabular}

\section{DISCUSIÓN}

La tomografía computarizada se ha utilizado para evaluar de manera tridimensional estructuras en el área maxilofacial que en las radiografías convencionales no son muy claras. La TCHC es un tipo de tomografía computarizada que permite una evaluación con bastante precisión de tejidos los duros y es utilizada comúnmente en odontología antes de procedimientos quirúrgicos ya que puede brindar un campo de visión pequeño, una imagen con alta resolución espacial y a la vez una menor dosis de radiación en comparación TEM $(4,12,13)$

En el presente estudio se evaluó al CNP en una población peruana a través de TCHC mediante cortes sagitales. En la muestra se encontró que el CNP mostró gran variabilidad con respecto a sus dimensiones y angulación así como en su morfología. Las diferentes formas del CNP que se observan en una vista sagital se deben a las distintas zonas de estrechamiento $\mathrm{u}$ obliteración que puede presentar el conducto $(1,14)$.

En el presente estudio la forma más común encontrada fue la cilíndrica con un total de 89 casos, seguida de la forma de embudo con un total de 38 casos, y las formas menos comunes fueron la forma de reloj de arena y de huso con 20 y 7 casos respectivamente. No hubo una diferencia en relación a la forma y al sexo de los pacientes, resultados equiparables a los encontrados en estudios realizados por Thakur (1), Liang (5), Mardinger (8), Mishra (9) Tözüm (10), Yaser (14), Nasseh (15) en donde la forma de CNP más encontrada en una vista sagital fue la forma cilíndrica.

En contraste con el presente estudio, Fukuda informó que la forma de embudo era la más común (16). 
Por otra parte los resultados informados por Thakur mostraron que en los pacientes de sexo masculino la forma más encontrada fue la de embudo, a diferencia con el presente estudio en el que la forma más encontrada tanto para el sexo masculino y femenino fue la cilíndrica (1). Por otra parte cabe resaltar que las formas de embudo y de reloj de arena presentan un foramen incisivo de mayor diámetro anteroposterior y por lo tanto estas formas relativamente menos frecuentes deben tenerse en cuenta a la hora de la realización de procedimientos quirúrgicos en esa área.

En esta investigación se reportó un promedio de longitud para el CNP de $10,83 \mathrm{~mm}( \pm 2,13)$, resultados muy cercanos a los reportados por Thakur (1)Y, and Z, Tözüm (10), Nasseh (15), Fukuda (16)(16 que informaron un promedio de $10,08 \mathrm{~mm}( \pm 2,25)$, $10,86 \mathrm{~mm}( \pm 2,67) 11,52 \mathrm{~mm}(7,20-17,40 \mathrm{~mm}), 11,75$ $\mathrm{mm}$ respectivamente. Por otro lado, el promedio de conductos más largos reportados fueron por los estudios de Hakbilen (2), Al-Amery (3), Kajan (12), y Panjnouch (17), con un promedio de $12,84 \mathrm{~mm}$ ( \pm $2,88), 13,65 \mathrm{~mm}( \pm 3,12)$ y $14,1 \mathrm{~mm}( \pm 3,0) 16,33$ $\mathrm{mm}( \pm 4,43) \mathrm{mm}$ respectivamente, mientras que el promedio más corto reportado fue por el estudio realizado por Mraiwa de $8,1 \mathrm{~mm}( \pm 3,4)(4)$. En el presente estudio los CNP en el sexo femenino fueron más cortos que en el sexo masculino, esto es coincidente con los estudios de Thakur (1)Y, and Z, Hakbilen (2), Mishra (9) y Tözüm (10). Este último resultado se atribuye a que el sexo masculino tiene relativamente dimensiones cráneo faciales más grandes en comparación con el sexo femenino (18). Por otra parte en un el estudio realizado por AlAmery (3) no hubo diferencia entre promedio del sexo masculino que fue de $17,96 \mathrm{~mm}( \pm 3,6)$ y el femenino que fue $17,96 \mathrm{~mm}( \pm 4,18)$.

El diámetro anteroposterior del foramen incisivo en el presente estudio varió desde $1,5 \mathrm{~mm}$ a $6,2 \mathrm{~mm}$ con un promedio de $3,44 \mathrm{~mm}( \pm 0,80)$; esta medida estuvo cercana respecto al estudio realizado por Liang (5) y el de Kajan (12), quienes obtuvieron un promedio de $3,44( \pm 0,9) \mathrm{mm}$ y $3,53( \pm 1,1) \mathrm{mm}$ correspondientemente. Sin embargo estos valores fueron más bajos que los estudios realizados por Maraiwa (4)morphology and dimensions of the nasopalatine canal on two-dimensional and threedimensional (2D and 3D, Salemi (19), y Nasseh (15) que obtuvieron un promedio de $4,3 \mathrm{~mm}( \pm 0,9), 4,74 \mathrm{~mm}$
$( \pm 1,11), 4,91 \mathrm{~mm}(2,5-7,30 \mathrm{~mm})$ respectivamente, pero superiores al estudio realizado por Al-Amery (3) que reportó un promedio de $2,80 \mathrm{~mm}( \pm 0,82)$. En el presente estudio no hubo una diferencia marcada en cuanto al diámetro anteroposterior del FI y el sexo, resultados similares a los encontrados por Thakur (1) Y, and Z, Hakbilen (2) y Nasseh (15). Algunos autores afirman que cuando un FI supera los $10 \mathrm{~mm}$, se deben considerar la presencia de condiciones patológicas (4)morphology and dimensions of the nasopalatine canal on two-dimensional and three-dimensional (2D and 3D. La gran variabilidad de medidas reportadas en la literatura tanto para la longitud el CNP como del diámetro anteroposterior del FI podría atribuirse a que los estudios fueron realizados en diferentes países y por ende las medidas antropométricas varían en relación a la raza $(1 \mathrm{Y}$, and Z,2,4,5morphology and dimensions of the nasopalatine canal on twodimensional and three-dimensional (2D and 3D,11).

En lo que respecta al grado de inclinación del CNP en relación a la perpendicular del plano palatino, el resultado promedio fue de 16,44 grados equiparable con el estudio de Nasseh (15) que fue de 17,09 grados. En relación al sexo en el presente estudio hubo una relativa diferencia, debido a que el promedio del sexo masculino fue menor con 15.08 grados que el femenino con 17,52 grados. La orientación del CNP fue definida si el ángulo resultante era mayor o igual a 10 grados como inclinado y si era inferior a 10 grados como vertical, Thakur encontró que el $51 \%$ de sus conductos estaban inclinados y el $49 \%$ eran verticales (1), a diferencia del presente estudio que obtuvo un $78,6 \%$ de conductos inclinados y un $21,4 \%$ de conductos verticales, lo cual muestra la gran variabilidad de orientación que puede tener el CNP. En cuanto a la relación entre orientación y sexo no hubo una marcada diferencia, siendo encontrados con mayor frecuencia los CNP inclinados para ambos, en concordancia con la investigación realizada por Thakur (1)Y, and Z. El grado de inclinación y orientación del CNP podría deberse al grado de desarrollo, forma o posición del maxilar superior, mientras menos desarrollado o más retruido se encuentre el maxilar, el grado de inclinación del CNP podría ser menor y por lo tanto tendría una orientación más vertical; así mismo en una relación esquelética intermaxilar Clase II el CNP podría presentarse más inclinado y por ende en una relación esquelética Clase III el CNP podría observarse más vertical (20). 
En esta investigación, existieron factores limitantes como: a) El grupo muestral de población peruana concierne solo a adultos lo que hace que los resultados no sean apropiados para generaciones más jóvenes y por lo tanto sería recomendable realizar investigaciones en este sentido. b) El tamaño muestral fue mediano, por lo que es esencial realizar estudios adicionales en una muestra más grande para obtener resultados más precisos y además sería recomendable establecer asociación entre diferentes variables. Finalmente también se recomienda la realización de más estudios concernientes a la inclinación del CNP en relación a la posición, forma y desarrollo del hueso maxilar para establecer si existe alguna relación.

Este estudio demostró la variabilidad del CNP en cuanto a la morfología, angulación, y el diámetro anteroposterior del FI en la población peruana. El sexo masculino mostró medidas mayores en longitud del CNP en comparación con el sexo femenino y a su vez el sexo femenino presentó una mayor angulación en relación al paladar duro. Se debe resaltar la importancia de un análisis minucioso a través de TCHC para la evaluación de este reparo anatómico ya que proporciona al odontólogo que se desempeña en las áreas de cirugía maxilofacial, periodoncia e implantología información valiosa para obtener una adecuada planificación quirúrgica en el maxilar anterior, minimizando así, posibles complicaciones en sus pacientes.

\section{Correspondencia:}

Jorge Luis Obando Castillo

Correo electrónico: jorge.obando@upch.pe

\section{REFERENCIAS BILIOGRÁFICAS}

1. Thakur AR, Burde K, Guttal K, Naikmasur VG. Anatomy and morphology of the nasopalatine canal using cone-beam computed tomography. Imaging Sci Dent. 2013;43(4):273-81.

2. Hakbilen S, Magat G. Evaluation of anatomical and morphological characteristics of the nasopalatine canal in a Turkish population by cone beam computerized tomography. Folia Morphol (Warsz). 2018;77(3):527-35.

3. Al-Amery SM, Nambiar P, Jamaludin M, John J, Ngeow WC. Cone beam computed tomography assessment of the maxillary incisive canal and foramen: Considerations of anatomical variations when placing immediate implants. PLoS One. 2015;10(2):1-16.

4. Mraiwa N, Jacobs R, Van Cleynenbreugel J, et al. The nasopalatine canal revisited using 2D and 3D CT imaging. Dentomaxillofacial Radiol. 2004;33(6):396402.

5. Liang X, Jacobs R, Martens W, et al. Macro-and micro-anatomical, histological and computed tomography scan characterization of the nasopalatine canal. J Clin Periodontol. 2009;36(7):598-603.

6. Kraut R, Boyden D. Location of incisive canal in relation to central incisor implants. Implant Dent. 1998;7(3):221-5.

7. Bornstein MM, Balsiger R, Sendi P, Von Arx T. Morphology of the nasopalatine canal and dental implant surgery: A radiographic analysis of 100 consecutive patients using limited cone-beam computed tomography. Clin Oral Implants Res. 2011;22(3):295-301.

8. Mardinger $\mathrm{O}$, Namani-Sadan $\mathrm{N}$, Chaushu G, Schwartz-Arad D. Morphologic Changes of the nasopalatine canal related to dental implantation: A radiologic study in different degrees of absorbed maxillae. J Periodontol. 2008;79(9):1659-62.

9. Mishra R, Thimmarasa VB, Jaju PP, Mishra R, Shrivastava $A$. Influence of gender and age on nasopalatine canal: A cone-beam computed tomography study. J Dent Implant. 2017;7(1):15-9.

10. Tözüm TF, Güncü GN, Yıldırım YD, et al. Evaluation of maxillary incisive canal characteristics related to dental implant treatment with computerized tomography: A clinical multicenter study. J Periodontol. 2012;83(3):337-43.

11. Song WC, Jo DI, Lee JY, et al. Microanatomy of the incisive canal using three-dimensional reconstruction of microCT images: An ex vivo study. Oral Surg Oral Med Oral Pathol Oral Radiol Endod. 2009;108(4):583-90.

12. Kajan ZD, Kia J, Motevasseli S RS. Evaluation of the nasopalatine canal with cone-beam computed tomography in an Iranian population. Dent Res J. 2015;12(1):14-9.

13. Naitoh M, Nakahara K, Suenaga Y. Comparison between cone-beam and multislice computed tomography depicting mandibular neurovascular canal. Oral Surg Oral Med Oral Pathol Oral Radiol Endod. 2010;109(1):e25-31.

14 Yaser S, Mahkameh M, Sepideh R, Mahtab K, Maryam E. Assessment of nasopalatine canal anatomic variations using cone beam computed tomography in a group of Iranian Population. 2017;14(1):1-9.

15. Nasseh I, Aoun G, Sokhn S. Assessment of the nasopalatine canal: An anatomical study. Acta Inform Medica. 2017;25(1):34-8.

16. Fukuda M, Matsunaga S, Odaka K, et al. Three-dimensional analysis of incisive canals in human dentulous and edentulous maxillary bones. Int J Implant 
Dent. 2015;1(1):12.

17. Panjnoush M, Norouzi H, Kheirandish Y, Shamshiri AR, Mofidi N. Evaluation of morphology and anatomical measurement of nasopalatine canal using cone beam computed tomography. J Dent. 2016;13(4):287-94.

18. Utsuno H, Kageyama T, Uchida K, et al. Establishment of a prediction method for the mid-facial region of unknown human Mongoloid skeletal remains. Forensic Sci Int. 2018; 288:297-303.

19. Salemi F, Moghadam F, Farhadian M. Three-dimensional assessment of the nasopalatine canal and the surrounding bone using cone-beam computed tomography. J Periodontal Implant Dent. 2016;8(1):1-7.

20. Laowansiri U, Behrents RG, Araujo E, Oliver DR. Maxillary growth and maturation during infancy and early childhood. 2013;83(4):563-71.

Recibido: 20-07-2019

Aceptado: 21-11-2019 